\title{
NUEVAS LOCALIDADES PARA GRETA DIAPHANUS QUISQUEYA (FOX, 1963) Y PAPILIO DEMOLEUS LINNAEUS, 1758 (LEPIDOPTERA: PAPILIONOIDEA) EN LA HISPANIOLA
}

\author{
Ruth H. Bastardo
}

Instituto de Investigaciones Botánicas y Zoológicas Prof. Rafael M. Moscoso. Universidad Autónoma de Santo Domingo, Santo Domingo, República Dominicana.r_bastardo@hotmail.com

\section{RESUMEN}

Se documenta por primera vez la presencia de las mariposas Greta diaphanus quisqueya (Fox, 1963) (Nymphalidae, Danainae) y Papilio demoleus Linnaeus, 1758 (Papilionidae) para Haití (isla Hispaniola). Nuevas localidades para ambas especies en República Dominicana también son incluidas. Cestrum sphaerocarpum O. E. Schulz y Cestrum inclusum Urb. (Solanaceae) se registran como nuevas plantas hospederas para las larvas de $G$. d. quisqueya.

Palabras clave: mariposas, Haití, República Dominicana, isla Hispaniola.

Title: New localities for Greta diaphanus quisqueya (Fox, 1963) and Papilio demoleus Linnaeus, 1758 (Lepidoptera: Papilionoidea) on Hispaniola.

\section{ABSTRACT}

The presence in Haiti (Hispaniola Island) of the butterflies Greta diaphanus quisqueya (Fox, 1963) (Nymphalidae, Danainae) and Papilio demoleus Linnaeus, 1758 (Papilionidae) is documented for the first time. New localities for both species in the Dominican Republic are also included. Cestrum sphaerocarpum O. E. Schulz y Cestrum inclusum Urb. (Solanaceae) are reported as new host plants for the larvae of $G$. $d$. quisqueya.

Key words: butterflies, Lepidoptera, Haiti, Dominican Republic, Hispaniola Island.

La isla Hispaniola es la segunda en tamaño de las Antillas Mayores. La porción occidental de la isla está ocupada por la República de Haití y la oriental por la República Dominicana. La fauna de lepidópteros diurnos (Lepidoptera: Papilionoidea y Hesperioidea) de ambos países es bastante similar, pero la República Dominicana, con dos tercios del territorio de la isla, una variedad de ambientes, incluyendo las montañas de mayor elevación en el Caribe y cobertura boscosa mucho mayor, tiene más especies de distribución restringida a su territorio aunque no necesariamente endémicas. El trabajo más reciente acerca de la fauna de la isla (Pérez Gelabert, 2008) registra alrededor de 200 especies. Sourakov y Zakharov (2011) reducen este número a unas 190 especies de acuerdo con la revisión del género Calisto Hübner, 1823 para La Hispaniola. A pesar del gran conocimiento de la fauna de mariposas de la isla que actualmente se tiene, Greta diaphanus quisqueya (Fox, 1963), subespecie endémica de República Dominicana, propia de las montañas, y Papilio demoleus Linnaeus, 1758, de reciente introducción en este país (Guerrero et al., 2004) pero con una distribución más amplia, no han sido registradas para Haití. Este trabajo documenta la presencia de ambas especies por primera vez para ese país; se presentan además nuevas localidades para ambas especies en la República Dominicana y nuevas plantas hospederas para G. d. quisqueya (Fig. 1).

1. Papilio demoleus (Lepidoptera, Papilionidae). Es una plaga de los cítricos de origen asiático, registrada recientemente para las Antillas a partir de ejemplares colectados en el 
extremo este de la República Dominicana (Guerrero et al., 2004) y para Puerto Rico (Homziak y Homziak, 2006); Lauranzón et al. (2011) refieren los registros hechos por Garraway y Murphy para Jamaica en el 2006 y Núñez para Cuba en el 2007. Todavía no se sabe como llegó esta especie a la República Dominicana, pero Eastwood et al. (2006) confirmaron que la población que se encuentra en el país se originó en el sureste de Asia y sugieren una única introducción en el 2004.

Durante una expedición realizada en febrero de 2006 a la Reserva de la Biosfera Macaya fueron vistos dos individuos, uno en el Aeropuerto Internacional de Les Cayes, libando en flores cultivadas de Zinnia sp. (Asteraceae) y el segundo en el pueblo costero de Port Salut, volando en los alrededores de algunas plantas de limón, Citrus $x$ aurantifolia (Christm.) Swingle (Fig. 2 A-C). Este es el primer registro sobre la presencia de esta plaga para Haití, aunque no se encontró en el área de influencia de la Reserva. El hallazgo de $P$. demoleus en Haití era de esperarse, pues se la considera como una especie invasora de rápida dispersión y así lo demuestran los registros cercanos en el tiempo para las otras islas de las Antillas Mayores. Su preferencia por las plantas de limón cultivadas y las flores de jardín ha tenido mucho que ver en esta rápida dispersión. En la República Dominicana, Walhlberg y Peña (2007) la registraron del Parque Nacional Jaragua, Pedernales, en el suroeste de la República Dominicana. Otras localidades fronterizas donde también se ha encontrado son el pueblo de Pedernales, en la provincia Independencia, camino a Sierra de Neiba y en Montecristi. En la provincia Hato Mayor se encontraron larvas en plantas de naranja, Citrus sinensis (L.) Osbeck, atacadas por la araña de los cítricos, Cyrtophora citricola (Forskål, 1775), otra especie invasora.

Localidades. Haití: Les Cayes, Aeropuerto Antoine-Simon, 18 Q 628080 E 2020605 N, 1.II.2006, R. Bastardo, un ejemplar fotografiado libando en flores de Zinnia sp. (Asteraceae). Les Cayes, Hotel Concord, 18 Q 631635 E 2011853 N, 85 msnm, 1.II.2006, R. Bastardo, un ejemplar en el área del jardín. Playa Port Salut, 18 Q 612250 E 2001295 N, 9 msnm, 11.II.2006, R. Bastardo, $P$. demoleus volando cerca de plantas de limón en el patio de una casa cerca de la playa. República Dominicana: Sierra de Neiba, Prov. Independencia, La Descubierta, carretera hacia Los Pinos del Edén, 19 Q 209106 E 2055199 N, 143 msnm, 31.VII.2005, S. Vélez, R. Bastardo, J. Henríquez, A. Marmolejo, zona de transición bosque seco-húmedo, un ejemplar observado. Prov. Independencia, La Descubierta, frente al hotel, calle Padre Billini, 19 Q 211696 E 2055317 N, 37 msnm, 31.VII.2005, S. Vélez, R. Bastardo, J. Henríquez, A. Marmolejo, bosque seco, un ejemplar observado. Prov. Bahoruco, Villa Jaragua, 1.VIII.2005, S. Vélez, R. Bastardo, J. Henríquez, A. Marmolejo, alredor de cinco ejemplares volando entre los patios de las casas. Cordillera Central, Prov. Santiago, Jacagua, 19 Q 321660 E 2157577 N, 206 msnm, 21.VIII.2005, R. Bastardo, bosque de transición seco-húmedo. Prov. Hermanas Mirabal, Reserva Científica La Salcedoa, La Jíbara, 631 msnm, 27/29.X.2011, R. Bastardo, R. Rodríguez, T. Clase, D. Jones, un ejemplar. Prov. Montecristi, Guayubín, Ranchadero, 19 Q 252487 E 2173847 N, 45 msnm, 25.XII.2005, R. Bastardo, en flores cerca de una casa, un ejemplar. Prov. Hato Mayor, en una finca cerca del pueblo, 19 Q 472719 E 2067662 N, 20.X.2007, R. Bastardo, larvas en diferentes instares.

2. Greta $d$. quisqueya (Nymphalidae: Danainae). Es la única de las mariposas de alas transparente (tribu Ithomini) que habita en La Hispaniola. Esta subespecie se distribuye en las principales cadenas montañosas de la República Dominicana: Sierra de Bahoruco, Sierra de Neiba, Cordillera Central y Cordillera Septentrional. Como otros miembros de esta tribu, Greta es una mariposa asociada a zonas ribereñas o ambientes muy húmedos en las montañas. Schwartz (1980) propuso que esta especie no se encontraba en Haití debido a la extensa deforestación existente en las montañas de ese país, dando como localidad más occidental los bosques nublados de Sierra de Bahoruco. Luego se consideró que la especie no estaba presente en Haití o 
que el hábitat necesario era muy raro y local (Gali y Schwartz, 1986). Schwartz (1987) tampoco la encontró en Sierra Martín García, considerando la ausencia de alturas adecuadas, humedad (lluvia) y pequeños cuerpos de agua como los factores que justificaban que no estuviera presente en esta localidad, pero sí en Sierra de Neiba.

Este representa el primer registro de G. d. quisqueya para Haití, ampliando así su distribución a toda la isla. Las nuevas localidades se encuentran en el extremo suroeste de la Península de Tiburón, en las elevadas montañas de Massif de la Hotte, en la misma región que otros investigadores visitaran décadas atrás. El lugar se encuentra subiendo hacia el Pic Le Ciel, a 1,484 msnm de elevación, una de las montañas que están camino a Pic Macaya dentro del Parque Nacional Pic Macaya. La vegetación propia de la zona es un pinar de Pinus occidentalis Sw. mezclado con vegetación latifoliada (Fig. 2 D-E). En el hábitat donde se observaron los adultos volando por primera vez hay una diminuta quebrada que sale de la montaña y en cuyos alrededores crecía Cestrum coelophlebium O. E. Schulz (Solanaceae), la planta hospedera, hasta el momento conocida, para G. d. quisqueya (Sourakov y Emmel, 1995). En este sitio, Greta fue mucho más abundante. La otra localidad se encuentra en la vertiente oeste de la misma loma, donde se observaron dos individuos volando en horas de la tarde y se colectó uno de ellos. Todos los ejemplares colectados se encuentran depositados en la colección entomológica del Instituto de Investigaciones Botánicas y Zoológicas de la Universidad Autónoma de Santo Domingo.

Nuevas localidades en la República Dominicana. En recientes expediciones se han localizado nuevas poblaciones de G. d. quisqueya en la República Dominicana. Una población encontrada en Sierra Martín García en el 2011 por Miguel Ángel Landestoy, en el bosque nublado a 1,343 msnm, representa el más reciente hallazgo. También hay una población nueva en la porción oriental de la Sierra de Bahoruco, en la Loma Pie de Palo a 1,303 msnm; nuevas poblaciones en Loma Los Guayuyos y en Loma Barbacoa $(2009,2010)$ amplían su distribución más hacia el sur en la Cordillera Central. Todas las poblaciones conocidas se encuentran en bosques nublados por encima de los 1,000 m de elevación o en cafetales bajo sombra a elevaciones menores (por encima de $800 \mathrm{msnm}$ ). Afortunadamente, la mayoría de las localidades históricas tomadas de Schwartz (1989) y los nuevos registros, se encuentran en sitios que son parte actualmente del Sistema Nacional de Áreas Protegidas de la República Dominicana. Esto aumenta las expectativas de conservación para este miembro singular de la fauna de la isla.

Nuevas plantas hospederas. Cestrum sphaerocarpum O. E. Schulz y Cestrum inclusum Urb., solanáceas endémicas de La Hispaniola, se registran como nuevas plantas hospederas para Greta d. quisqueya. Las observaciones ocurrieron en la Loma Barbacoa y en la Loma Los Guayuyos, áreas protegidas en la Cordillera Central de la República Dominicana. La utilización de $C$. sphaerocarpum se observó por primera vez en agosto de 2009 en la Loma Los Guayuyos, un sitio con remanentes de vegetación típica de bosque nublado; se observó una hembra ovipositando en el envés de las hojas (Fig. 2 F). En esta localidad, C. sphaerocarpum es frecuente en los caminos dentro del bosque. Esta planta alcanza unos $50 \mathrm{~cm}$ de alto y tiene flores vistosas amarillas (Fig. 2 G). En la Loma Barbacoa, se han observado en distintos momentos tanto la puesta de huevos como larvas de los primeros estadíos en el follaje joven (envés) de C. sphaerocarpum (cinco larvas) en octubre 2011. En esta misma zona y fecha también se encontraron larvas de los primeros instares en C. inclusum (23) y huevos (30) en C. coelophlebium (Fig. 2 H-J). Las larvas mostraron la conducta gregaria descrita para la especie (Sourakov y Emmel, 1995). En todos los casos, las plantas crecían en el camino dentro del bosque nublado lejos de cuerpos de agua. En Loma Barbacoa, $C$. sphaerocarpum y $C$. inclusum son más abundantes que $C$. coelophlebium. Sin duda alguna, la presencia de las plantas hospederas de las larvas debe considerarse un factor fundamental en la distribución de $G$. d. quisqueya y cualquier otra especie monofágica como esta. Las tres especies de Cestrum son propias de montañas. Es probable que la búsqueda en 


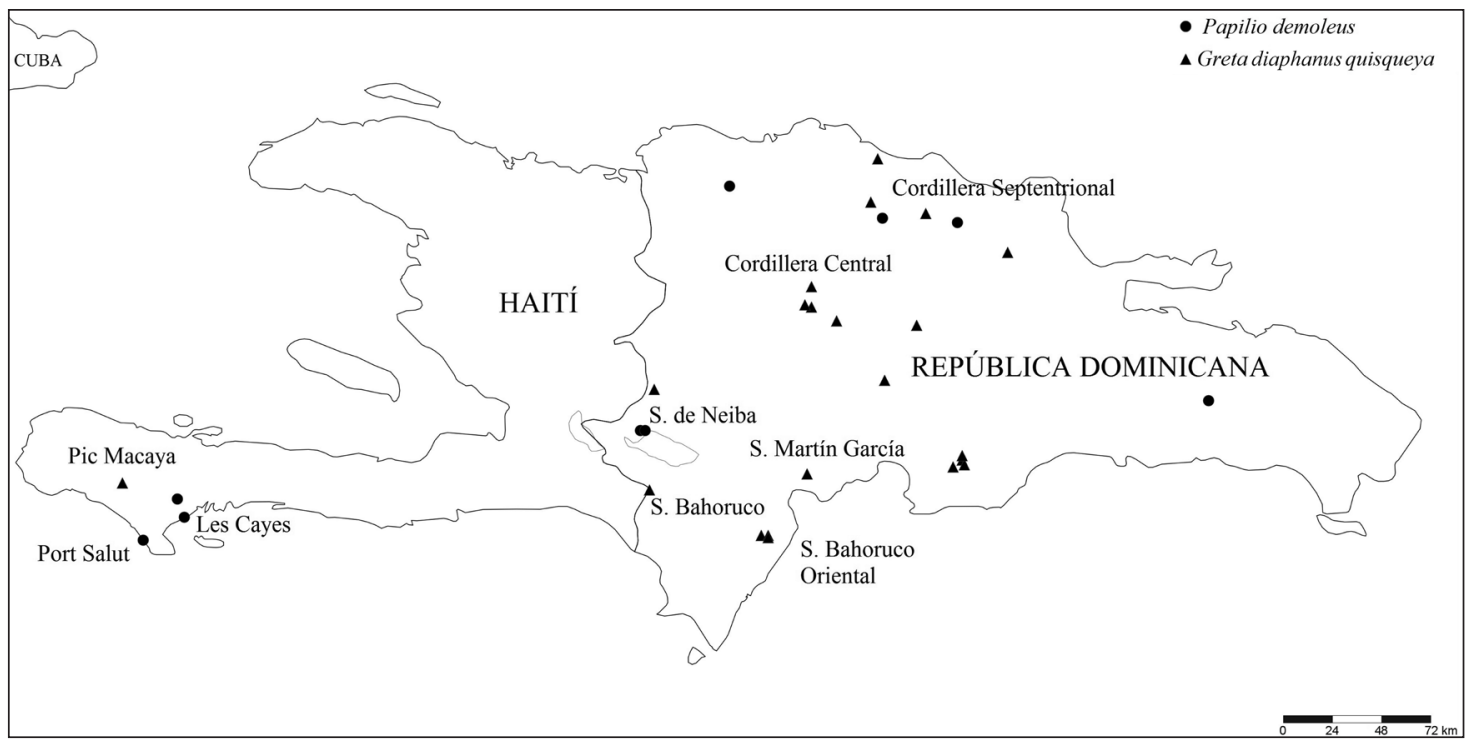

Figura 1. Distribución de Papilio demoleus y Greta diaphanus quisqueya en La Hispaniola.

otras localidades con altura adecuada (por encima de $600 \mathrm{msnm}$ ) y vegetación propia de bosque nublado o remanentes del mismo, incluyendo las plantas hospederas, produzcan el hallazgo de más poblaciones de esta especie.

Localidades. Haití: Reserva de la Biosfera Pic Macaya, Massif de la Hotte, Department du Sud, Trayecto Formon-Pic Le Ciel, 18 Q 603289 E 2028515 N, 1,484 msnm, 7.II.2006, R. Bastardo, B. Peguero, T. Clase; casi $50 \mathrm{~m}$ de una quebrada, bosque de pinos con latifoliadas, se observó libando en Eupatorium sp., seis ejemplares; Camino a Pic Le Ciel, subida por la vertiente oeste, 8.II.2006, más deforestada, dos individuos observados, uno capturado. República Dominicana: Sierra de Bahoruco (Parque Nacional): Prov. Independencia, Arriba de Puerto Escondido, Zapotén, borde del bosque nublado posteriormente destruido, 19 Q 213585 E $2026527 \mathrm{~N}$, 1,635 msnm, 18.X.2007, M. A. Landestoy. Prov. Barahona, Polo, Cortico, al frente de la charca, remanente de bosque nublado, impactado por ganadería y agricultura, 19 Q 264711 E 2003864 N, 1,376 m, 29.VII.2005, S. Vélez, J. Henríquez, A. Marmolejo, R. Bastardo, 1 vista. Prov. Barahona, Monumento Natural Miguel Domingo Fuertes, Loma Pie de Palo, 19 Q 267951 E 2003858 N, 1,303 msnm, 17.VI.2010, R. Bastardo, A. Espinosa, C. Suriel, S. Navarro, J. D. Hernández, bosque nublado, un ejemplar observado; Primer Manaclar,19 Q 268494 E 2002732 N, 1,215 msnm, 7.VIII.2010, R. Bastardo, un ejemplar observado. Sierra de Neiba, Prov. Elías Piña, cerca de Comendador (Schwartz, 1989), Coordenadas de Google Earth 19 Q 216059 E 2075656 N. Sierra Martín García: Prov. Azua, en la cima, bosque nublado cerca de antena en "El Alto de la Bandera", 19 Q 286068 E 2033459 N, 1,345 msnm, 14.XI.2011, M. A. Landestoy, D. P. Scantlebury, posado en una rama aproximadamente a $2 \mathrm{~m}$ sobre el suelo, 20:21 h, no cuerpos de agua, un ejemplar. Cordillera Central: Prov. Peravia, Reserva Científica Loma Barbacoa, subiendo desde Cañaveral hasta Loma Barbacoa, 19 Q 358745 E 2039733 N, 1,356 msnm, una observada. Localidad anterior, 19 Q 358705 E 2039811 N, 1,372 msnm, 4.VI.2010, R. Bastardo, A. Espinosa, C. Suriel, S. Navarro, J. D. Hernández, tres observadas; Prov. Peravia, Reserva Científica Loma Barbacoa, bajando desde Cañaveral hacia Las Yayitas, en cafetal bajo sombra, 19 Q 359046 E 2037696 N, 855 msnm, 5.VI.2010, los mismos colectores anteriores. Prov. Peravia, Reserva Científica Loma Barbacoa, La Monteada de Javier, 19 Q 358831 E 2042028 N, 1,288 msnm, 14/18.VII.2010, R. Bastardo, A. Espinosa, C. Suriel, S. Navarro, J. D. Hernández, bosque nublado, en el camino, cinco ejemplares observados; Loma Barbacoa, bosque nublado, 

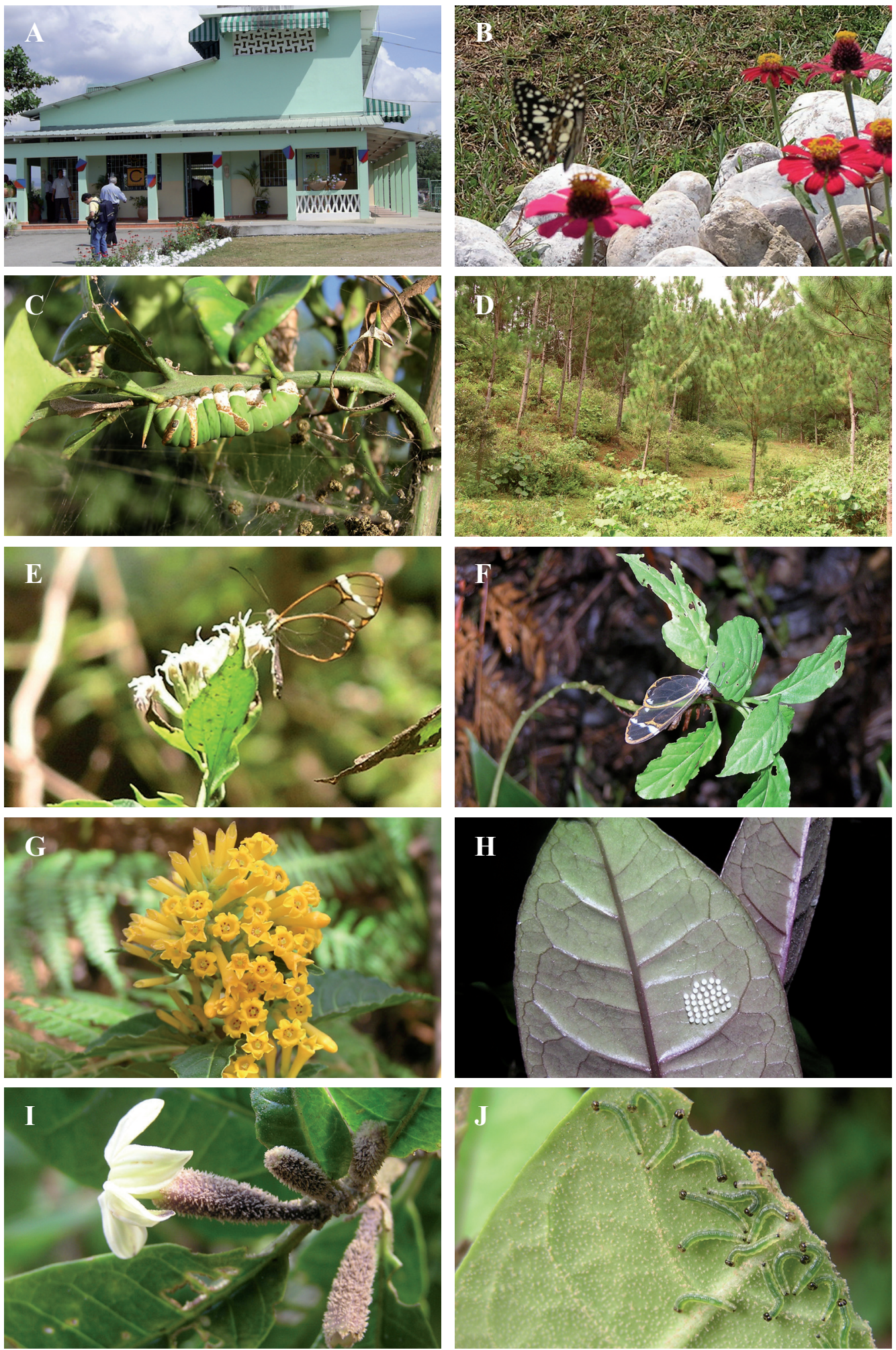

Figura 2. Papilio demoleus (A-C) y Greta diaphanus quisqueya (D-J). A, aeropuerto Les Cayes, Haití (foto T. Clase, 2006). B, en flores de Zinnia sp. en el aeropuerto. C, larva del último instar en planta de limón, prov. Hato Mayor, República Dominicana. D, pinar en Pic Le Ciel, Haití. E, en flores de Eupatorium sp. F, hembra ovipositando en C. sphaerocarpum. G, C. sphaerocarpum en flor. $\mathrm{H}$, huevos en $C$. coelophlebium.I, flores de $C$. inclusum. J, larvas en $C$. inclusum. 
19 Q 358487 E 2041611 N, 1,290 msnm, 29.IX/4X.2011, R. Bastardo, un adulto libando en Eupatorium sp.; cinco larvas en $C$. sphaerocarpum, 23 larvas de los primeros instares en $C$. inclusum. Prov. Peravia, Parque Nacional Luis Quinn, Loma Los Guayuyos, bosque nublado, 19 Q 354061 E 2036716 N, 1,334 msnm, 28.VIII.2009, R. Bastardo, A. Hilario, dos ejemplares observados, incluyendo una hembra poniendo huevos en C. sphaerocarpum. Reserva Científica Ébano Verde, Prov. La Vega, El Arroyazo, 19 Q 337594 E 2105122 N, 980 msnm, 5.VI.2005, R. Bastardo, E. Fernández, U. Borrell, un ejemplar observado. Parque Nacional Armando Bermúdez, Prov. La Vega, Manabao, La Ciénaga, camino a Los Tablones, 20.VIII.2006, D. Pérez, R. Bastardo, B. Hierro, un ejemplar observado. Prov. Santiago, Mun. San José de las Matas, Río Antonsape Malo (Schwartz, 1989); Juncalito, Río de los Negros, Cañada del Lechón, 19 Q 288888 E 2114615 N, 1,178 msnm, 8. IV.1999, R. Bastardo, cinco ejemplares observados, un ejemplar colectado; La Guácara, 19 Q 286055 E 2115254 N, 1,140 msnm, trayecto caseta en Loma del Oro hasta la caseta en La Guácara, 22.IV.1999, R. Bastardo, dos ejemplares colectados; Valle de Bao, 19 Q 286526 E 2107511 N, 1,860 msnm, R. Bastardo, un ejemplar colectado; Cordillera Septentrional: Prov. San Francisco de Macorís, Reserva Científica Loma Quita Espuela, firme de Loma Quita Espuela, 19 Q 379335 E 2140142 N, 715 msnm, 3/4.XII. 2003, D. Pérez, R. Bastardo, A. Marmolejo. Prov. Santiago (Monumento Natural) Pico Diego de Ocampo, 909-1,026 msnm (Schwartz, 1989); Prov. Puerto Plata (Monumento Natural) Mt. Isabel de Torres (Schwartz, 1989); Espaillat, 20 km SW Jamao al Norte, 793 msnm (Schwartz, 1989).

\section{AGRADECIMIENTOS}

Agradezco a Eladio Fernández y la Sociedad Audubon Haití por proveer los fondos y facilitar toda la logística necesaria para la realización de la expedición a la Reserva de la Biosfera Macaya, Haití. El Ministerio de Educación Superior, Ciencia y Tecnología facilitó los fondos necesarios para la realización de expediciones a la Cordillera Central y Sierra de Bahoruco Oriental (FONDOCYT No. 2008-2-C2-040 y No. 2009-102). El Dr. Daniel PérezGelabert (Smithsonian Institution, Washington, DC) hizo sugerencias valiosas al manuscrito. Teodoro Clase permitió el uso de la fotografía del aeropuerto de Les Cayes.

\section{LITERAURA CITADA}

Eastwood, R., S. Boyce y B. D. Farrell. 2006. The provenance of Old World swallowtail butterflies, Papilio demoleus (Lepidoptera: Papilionidae), recently discovered in the New World. Annals of the Entomological Society of America, 99: 164-168.

Gali, F. y A. Schwartz. 1986. The butterflies (Lepidoptera: Rhopalocera) of Morne La Visite and Pic Macaya, Haiti. US Agency for International Development, Gainesville, Florida, 16 pp.

Guerrero, K. A., D. Veloz, S. L. Boyce y B. Farrell. 2004. First New World documentation of an Old World citrus pest, the Lime Swallowtail Papilio demoleus (Lepidoptera: Papilionidae), in the Dominican Republic (Hispaniola). American Entomologist, 50: 227-229.

Homziak, N.T. y J. Homziak. 2006. Papilio demoleus (Lepidoptera: Papilionidae): A new record for The United States, Commonwealth of Puerto Rico. Florida Entomologist, 89: 485-488.

Lauranzón Meléndez, B., G. Gulli, J. Reyes Brea, M. del C. Fagilde y L. O. Melián Hernández. 2011. Observaciones sobre Papilio demoleus (Lepidoptera: Papilionidae), una especie invasora en Cuba. Solenodon, 9: 81-87. 
Pérez-Gelabert, D. E. 2008. Arthropods of Hispaniola (Dominican Republic and Haiti): a checklist and bibliography. Zootaxa, 1831: 1-530.

Schwartz, A. 1980. Deforestación y Rhopalocera una comparación en la recolección de mariposas entre República Dominicana y Haití. Naturalista Postal, 30/80.

Schwartz, A. 1987. Butterflies of the Sierra Martin Garcia, Republica Dominicana. Caribbean Journal of Science, 23: 418-431.

Schwartz, A. 1989. The butterflies of Hispaniola, University of Florida Press, Gainesville, 580 pp.

Sourakov, A. y E. V. Zakharov. 2011. "Darwin's butterflies"? DNA barcoding and the radiation of the endemic Caribbean butterfly genus Calisto (Lepidoptera, Nymphalidae, Satyrinae). Comparative Cytogenetics, 5: 191-210.

Sourakov, A. y T. C. Emmel. 1995. Life History of Greta diaphana from the Dominican Republic (Lepidoptera: Nymphalidae). Tropical Lepidoptera, 6: 155-157.

Walhlberg, N. y C. Peña. 2007. A Dominican Republic sojourn. News of the Lepidopterists' Society, 49: 12-15.

[Recibido: 25 de abril, 2012. Aceptado para publicación: 18 de mayo, 2012] 